\title{
XPS Studies of Some Chemically Synthesized Polypyrrole-Organic Acceptor Complexes
}

\author{
E. T. KANG, H. C. TI, and K. G. NeOH \\ Department of Chemical Engineering, National University of Singapore, \\ Kent Ridge, Singapore 0511
}

(Received May 30, 1988)

\begin{abstract}
X-Ray photoelectron spectroscopy (XPS) studies have been performed on polypyrrole-2,3-dichloro-5,6-dicyano-p-benzoquinone (PPY-DDQ) and polypyrrole-tetrachloro$o$-benzoquinone (PPY-chloranil) complexes prepared via the simultaneous chemical polymerization and oxidation of pyrrole by the respective acceptor. XPS data suggested that the charge transfer interactions in both complexes probably proceeded further than the pure formation of a molecular complex. The negative charges of both organic anions were mainly localized on the oxygen and the chlorine atoms and to some extent on the nitrogen of DDQ. More extensive charge transfer interaction and higher fraction of pyrrole nitrogen with positive charge were observed in the PPY-DDQ complex, which exhibited a substantially higher electrical conductivity than the corresponding PPY-chloranil complex.

KEY WORDS XPS / Polypyrrole / 2,3-Dichloro-5,6-dicyano-p-benzoquinone / Chloranil / Charge Transfer Interactions /
\end{abstract}

Due to their relative physicochemical and electrical stability and well-characterized stoichiometry, highly conductive polyheterocycles, polypyrrole (PPY) in particular, have attracted a great deal of interest in recent years. ${ }^{1,2}$ Most of the recent interest on conductive PPYs, however, are devoted to the materials obtained from electrochemical polymerization and oxidation. For example, most of the X-Ray photoelectron spectroscopy (XPS) studies were centered on electrochemically prepared PPYs and related polyheterocycles. ${ }^{3-7}$ We reported recently on the XPS technique as a convenient tool for analysing the polymer-acceptor interactions in chemically synthesized PPY-halogen complexes. ${ }^{8}$

XPS technique has also been found to be suitable for studying electron donor-acceptor interactions in organic systems because of its ability to elucidate the structure of reaction products and to detect any redistribution of charges within the molecules resulting from charge transfer. ${ }^{9}$ Although numerous charge transfer interactions between polymers and various organic electron acceptors have been reported, ${ }^{10-13}$ the application of XPS technique in the studying of polymer-organic acceptor interactions has been limited. Recently, we reported on the simultaneous chemical polymerization and oxidation of pyrrole by organic electron acceptors, 2,3-dichloro-5,6dicyano- $p$-benzoquinone (DDQ) and tetrachloro-o-benzoquinone (chloranil). ${ }^{14}$ Thus, it would be of great interest to carry out a detailed XPS study on this new family of chemically synthesized PPY-organic acceptor complexes. It is hoped that the results obtained therefrom may help to provide a better understanding of the charge transfer interactions in these complexes. 


\section{EXPERIMENTAL}

\section{Polymer Samples}

The polymer samples used for this study comprise of PPY-DDQ and PPY-chloranil complexes. All samples were prepared in the bulk via the simultaneous chemical polymerization and oxidation of pyrrole by the respective organic acceptor. ${ }^{14}$ The PPY-DDQ and PPY-chloranil complexes so prepared are granular in nature and have room temperature electrical conductivity in the order of $10^{-1}$ and $10^{-3} \mathrm{~S} \mathrm{~cm}^{-1}$, respectively, when measured on compressed pellets. Conductivities below room temperature were measured in situ in a liquidnitrogen cryostat in the presence of an inert atmosphere. The conductivity of both complexes exhibits only a weak temperature dependence at temperatures below room temperature, as shown in Figure 1, in the conventional two probe measurements. The $1 / 4$ power temperature dependence of conductivity has also been widely observed in other chemically ${ }^{15}$ as well as electrochemically ${ }^{16}$ synthesized and oxidized PPYs and has been attributed to the presence of Mott's model ${ }^{17}$ of variable-range hopping between localized states near the Fermi surface. The infrared absorption spectra for both complexes suggest the presence of a linear chain of $\alpha, \alpha^{\prime}$-disubstituted pyrrole rings. ${ }^{14}$

\section{XPS Measurements}

X-Ray photoelectron spectroscopy (XPS) measurements were made on an ESCA 750 spectrometer with a $\mathrm{MgK \alpha} \mathrm{X}$-Ray source (1253.6 eV photons) through the courtesy of Toray Research Center, Inc., Shiga, Japan. All samples were measured in powder form. The sample sizes were in the order of 1 to $10 \mathrm{~mm}$ in diameter. All spectra were referenced to the $\mathrm{C} 1$ s neutral carbon peak at $284.6 \mathrm{eV}$. The peak area ratios for various elements were corrected by experimentally determined instrumental sensitivity factors.

\section{RESULTS AND DISCUSSION}

Since both PPY-DDQ and PPY-chloranil complexes contain oxygen, nitrogen, chlorine and carbon, the core-level spectra were obtained for all of the four elements. Figure $2 \mathrm{a}$

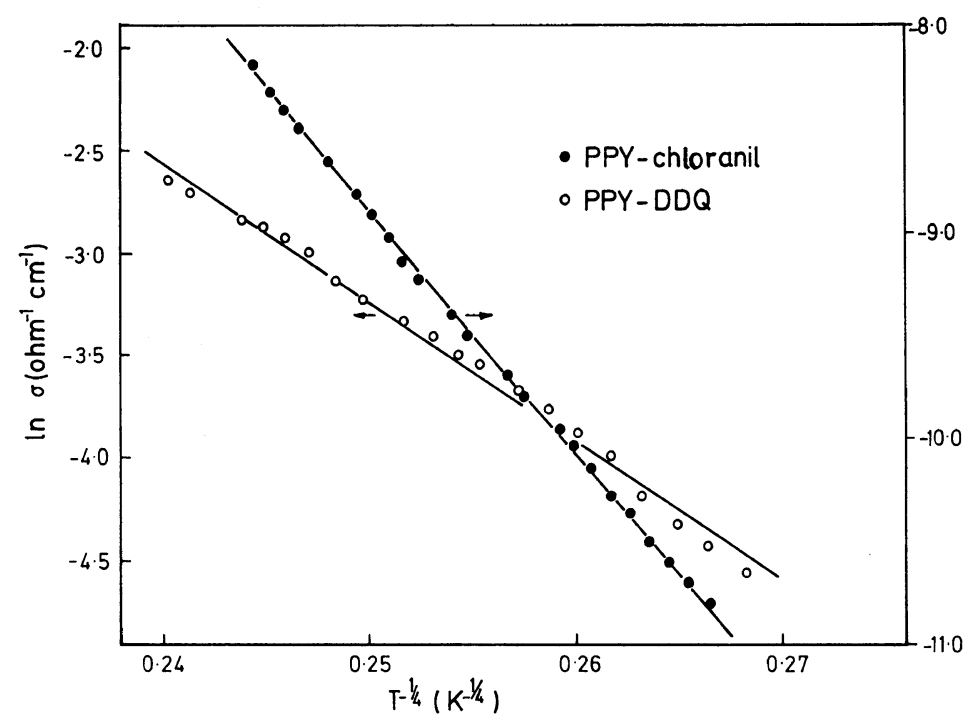

Figure 1. Temperature dependence of the electrical conductivity of PPY-DDQ and PPY-chloranil complexes. 
and $2 \mathrm{~b}$ show the respecive N1s core-level spectrum for the PPY-DDQ and PPYchloranil complex. Both spectra were fitted to Gaussian component peaks. For both complexes, the main component peak at about $399.5 \mathrm{eV}$ is attributable to the neutral nitrogen species of the complex. The presence of a small component peak in the PPY-DDQ complex which has a chemical shift of $-1.5 \mathrm{eV}$ from the neutral nitrogen peak can probably be associated with the presence of negatively charged nitrogen in the organic acceptor. A main N1s peak at about $398 \mathrm{eV}$ has been reported for the organic salt complexes involving tetracyanoquinodimethan anion $\left(\mathrm{TCNQ}^{-}\right) .{ }^{18}$ The two high binding energy components at $401 \mathrm{eV}$ and $402.5 \mathrm{eV}$ can be assigned to nitrogen atoms of the pyrrole associated with

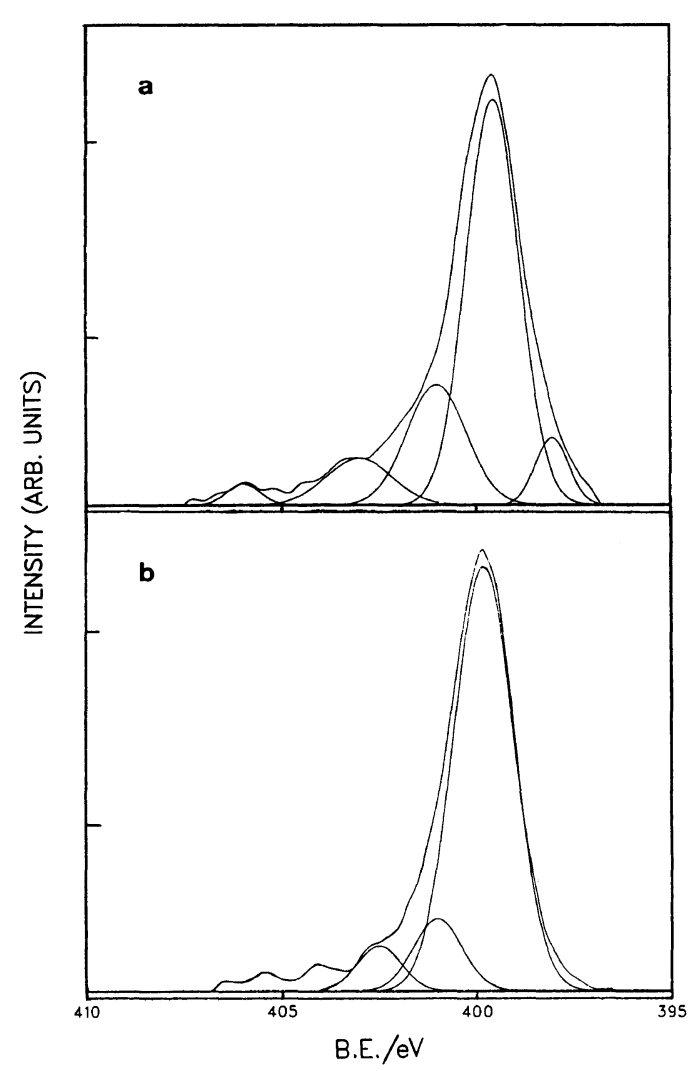

Figure 2. N1s XPS core-level spectrum of (a) PPYDDQ and (b) PPY-chloranil complex. positive charge. ${ }^{7}$ The fraction of pyrrole nitrogen with positive charge was calculated to be about 0.18 for the PPY-chloranil complex and about 0.43 for the PPY-DDQ complex. The N1s spectrum of the latter complex has to be corrected for the contribution from the cyano group of DDQ. The DDQ acceptor level in this case can be readily determined from chlorine balance based on the corrected $\mathrm{C} 12 \mathrm{p}$ core-level peak area.

Figure $3 a$ and $3 b$ show the $C 12 p$ core-level spectrum for the PPY-DDQ and PPYchloranil complex, respectively. The spectrum of each complex can be deconvoluted into two major components with a shake-up satellite at the high binding energy tail. The component at

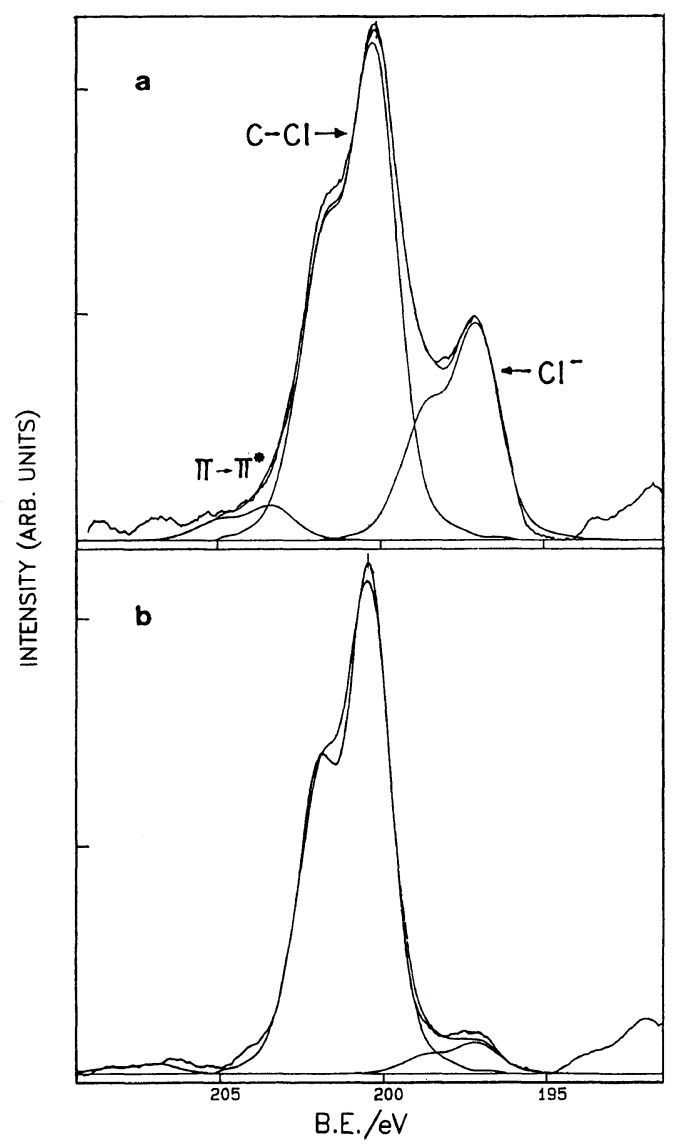

Figure 3. $\mathrm{Cl} 2 \mathrm{p}$ XPS core-level spectrum of (a) PPYDDQ and (b) PPY-chloranil complex. 
$197.2 \mathrm{eV}$ is attributable to chloride anion while the component at a higher binding energy of $200.5 \mathrm{eV}$ corresponds to covalently bonded chlorine. Comparison of the two spectra clearly shows that the proportion of chloride ion is substantially higher in the PPY-DDQ complex. The presence of shake-up satellite structure in both complexes indicates that the ring structure of the acceptor probably remains intact. However, the presence of chloride anion readily suggests that some of the chlorine atoms on the acceptor have been replaced probably by the formation of linkage between positively charged pyrrole nitrogen and the acceptor. The mechanism of interaction is supported by the overall charge balances

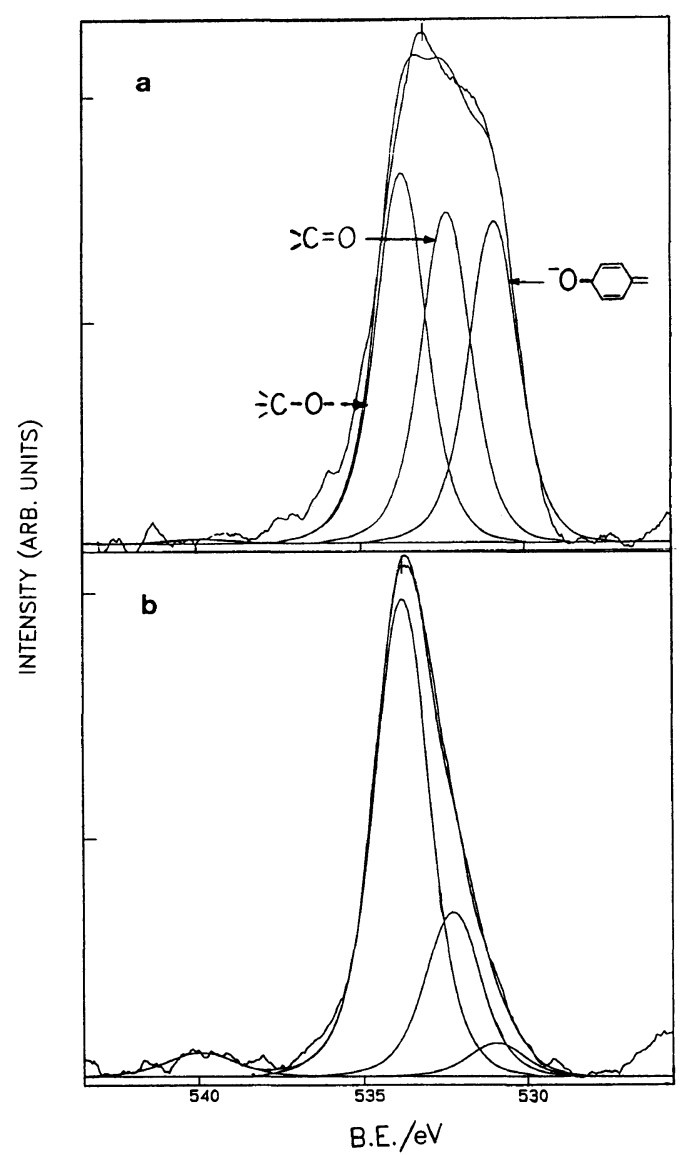

Figure 4. C1s XPS core-level spectrum of (a) PPYDDQ and (b) PPY-chloranil complex. of the complexes (see below). A similar mechanism has been proposed to account for the formation of chloride anion and pyridinium cation in the pyridine-chloranil charge transfer interaction. ${ }^{9}$

The $\mathrm{C}$ 1s core-level spectral for both complexes are shown in Figure $4 \mathrm{a}$ and $4 \mathrm{~b}$. The spectrum for each complex suggests the presence of three types of oxygen. The peak at $532.4 \mathrm{eV}$ can be assigned to the carbonyl structure of the original acceptor. A negative binding energy shift of $1.5 \mathrm{eV}$ probably corresponds to the formation of benzoquinone or semi-benzoquinone anion resulting from the charge transfer. ${ }^{9}$ The formation of DDQ and chloranil benzoquinone anion as a result of charge transfer has been widely observed..$^{10} \mathrm{On}$ the other hand, however, a positive binding energy shift of $1.5 \mathrm{eV}$ strongly suggests that some of the carbonyl oxygen are involved in the formation of $=\mathrm{C}-\mathrm{O}-$ structure. For PPY-chloranil complex, we may have to rule out any substantial formation of linkage between carbonyl oxygen and positively charged nitrogen of the pyrrole moiety, since the process would probably require equal amount of oxygen with positive and negative shift in binding energy to be observed. ${ }^{9}$ In the case of PPY-DDQ complex, about equal amount of oxgen with positive and negative shift in binding energy was observed. Surface oxidation ${ }^{8,19}$ products may also contribute to a small extent to the two higher binding energy species since the present complexes appear to contain a slight excess of oxygen, as in the case of other PPY complexes. ${ }^{19,20}$ In the present work, the ratio of the total area, after correction with the appropriate elemental sensitivity factor, of the positively-charged nitrogen (B.E. $\geqslant 401 \mathrm{eV}$ ) to the sum of benzoquinone anion (B.E. $531 \mathrm{eV}$ ), chloride anion (B.E. 197eV) and, in the case of PPY-DDQ complex, cyano anion (B.E. $398 \mathrm{eV}$ ) was calculated to be 0.95 and 1.10 , respectively, for the PPY-DDQ and PPY-chloranil complex. Thus, a close balance in the positive and negative charges was ob- 


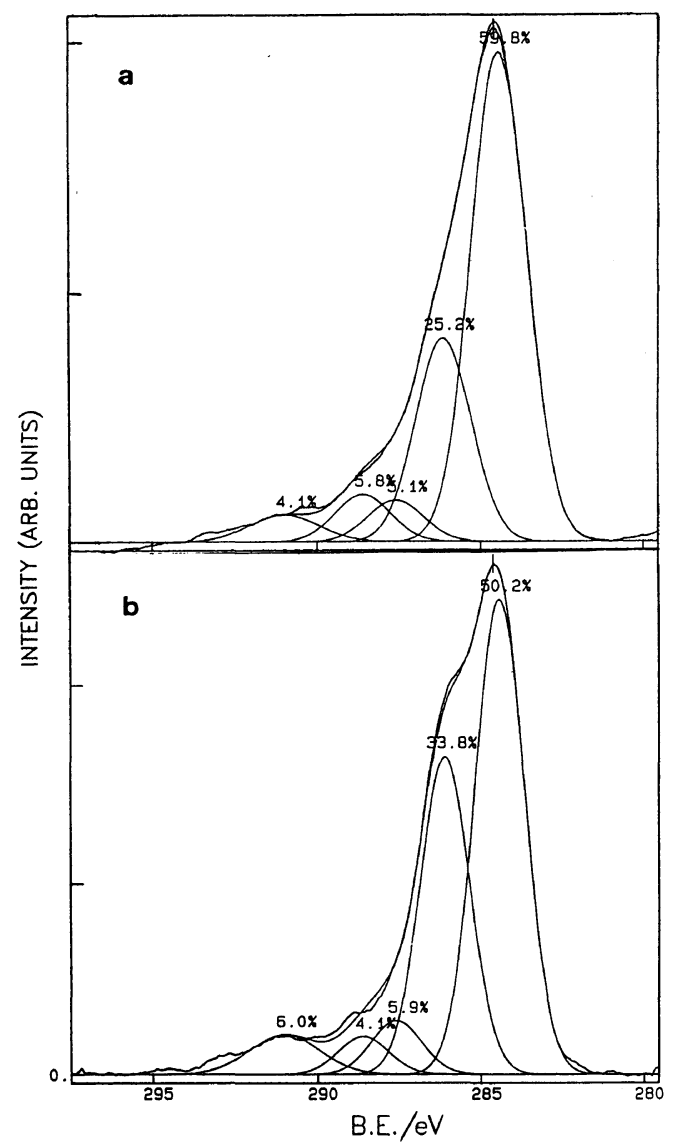

Figure 5. C1s XPS core-level spectrum of (a) PPYDDQ and (b) PPY-chloranil complex.

served in both complexes, based on the XPS results and the peak assignments.

The C1s core-level spectrum (Figure $5 \mathrm{a}$ and $5 b)$ of both complexes are skewed towards the high binding energy side with the main component peak at $284.4 \mathrm{eV}$. Similar line shape has been observed in other oxidized PPYs prepared electrochemically ${ }^{6}$ or chemically. ${ }^{8}$ The peak widths (FWHM) for the main component are $2.0 \mathrm{eV}$ and $1.8 \mathrm{eV}$, respectively, for the PPY-DDQ and PPY-chloranil complex. The broader peak observed in the former is probably due to the contribution from the cyano group of DDQ. In the PPY-chloranil complex, most of the chloranil contain the $\doteq \mathrm{C}-\mathrm{O}-$ structure and most of the chlorine remain covalently bonded to the acceptor. Thus, it is not surprising that the Cls corelevel spectra of the PPY-chloranil complex shows a significantly higher proportion of the peak component at $286.1 \mathrm{eV}$, attributable to the $\mathrm{C}-\mathrm{O}, \mathrm{C}-\mathrm{N}$, and $\mathrm{C}-\mathrm{Cl}$ linkages. The peak at $287.6 \mathrm{eV}$ is associated with the carbonyl group. However, the small peak at about $288.5 \mathrm{eV}$ is as yet unidentified, but the high binding energy suggests the presence of electron-withdraw atoms, possibly as a result of surface oxidation. ${ }^{8}$ Finally, at $291.0 \mathrm{eV}$, a shake-up satellite arising from the aromaticity of the polymer and acceptor can be resolved.

The normalized relative spectra intensities of the various elemental species suggest that there are 3-4 pyrrole units to every acceptor molecule. The result is consistent with the bulk elemental analysis, as well as with the stoichiometry of other oxidized PPYs prepared either by electrochemical ${ }^{2}$ or chemical $^{15,21}$ methods. The question concerning the exact chemical structure of the complexes cannot be answered without further experimental data, such as the initial position of attack by the pyrrole ring and the position of chlorine substitution. Furthermore, the valency of the acceptor anions needs special consideration. In the case of PPY-DDQ complex, the XPS data suggest that the total number of negative charges exceeds the total number of DDQ species in the complex. Thus, it is likely that some of the DDQ may exist as di- or higher valent anions. However, in the case of PPYchloranil complex, the total number of anions is lower than the number of chloranil species present. This suggests a mixed valence complex $^{22}$ with incomplete charge transfer. The result is in agreement with the earlier experimental observation that chloranil is less tightly complexed with PPY than DDQ ${ }^{14}$ However, one still cannot rule out the presence of di- and higher valent complexes, especially in the light that the presence of divalent anion has been suggested for the pyridine-chloranil complexes $^{9}$ and other complexes involving 
chloranil. $^{23}$

\section{CONCLUSION}

The nature of charge transfer interactions in chemically synthesized PPY-DDQ and PPYchloranil complexes were studied by XPS. More extensive charge transfer interactions were observed in the PPY-DDQ complexes, which exhibits a substantially higher conductivity. In both complexes, the negative charges were localized on the functional groups of the acceptor with the charge transfer having proceeded further than the formation of a molecular complex. Close balance was observed between the number of positively charged nitrogen and the sum of the total anion species in each complex.

Acknowledgements. The authors wish to thank Prof. T. Higashimura and Prof. T. Masuda of the Department of Polymer Chemistry, Kyoto University for their arrangement for the XPS measurements. One of us (ETK) acknowledges the financial support from Japan International Cooperation Agency for participation in the Japan-Singapore Technical Cooperation Program.

\section{REFERENCES}

1. See for example, "Handbook of Conducting Polymers," Vols. I and II, T. Skotheim, Ed., Marcel Dekker, New York, N. Y., 1986.

2. A. F. Diaz and K. K. Kanazawa, "Extented Linear Chain Compounds," Vol. 3, J. S. Miller, Ed., Plenum
Press, New York, N. Y., 1983.

3. W. R. Salaneck, R. Erlandsson, J. Prejza, I. Lundstron, and O. Inganess, Synth. Metals, 5, 125 (1983).

4. P. Pfluger and G. B. Street, J. Chem. Phys., 80, 544 (1984).

5. R. Erlandsson, O. Inganas, I. Lundstrom, and W. R. Salaneck, Synth. Metals, 10, 303 (1985).

6. T. A. Skotheim, M. I. Florit, A. Melo, and W. E. O'Grandy, Phys. Rev. B, 30, 484 (1984).

7. J. G. Eavas, H. S. Munro, and D. Parker, Polym. Commun., 28, 38 (1987).

8. E. T. Kang, H. C. Ti, K. G. Neoh, and T. C. Tan, Polym. J., 20, 399 (1988).

9. K. T. Ng and D. M. Hercules, J. Am. Chem. Soc., 97, 4168 (1975).

10. See for example, R. Foster, "Organic ChargeTransfer Complexes," Academic Press, New York, N. Y., 1969.

11. H. Hoegl, J. Phys. Chem., 69, 755 (1965).

12. T. Tsuitsui and S. Saito, Rep. Prog. Polym. Phys. Jpn., 25, 435 (1982).

13. Y. Kuwane, T. Masuda, and T. Higashimura, Polym. J., 12, 387 (1980).

14. E. T. Kang, K. G. Neoh, T. C. Tan, and Y. K. Ong, J. Polym. Sci., Polym. Chem. Ed., 25, 2143 (1987).

15. E. T. Kang, K. G. Neoh, and H. C. Ti, Solid State Commun., 60, 457 (1986).

16. A. Watanabe, M. Tanaka, and J. Tanaka, Bull. Chem. Soc. Jpn., 54, 2278 (1981).

17. N. F. Mott and E. A. Davis, "Electronic Processes in Non-crystalline Materials," Clarendon Press, 2nd ed, Oxford, 1979, p 34.

18. I. Ikemoto, M. Yamada, T. Sugano, and H. Kuroda, Bull. Chem. Soc. Jpn., 53, 1871 (1980).

19. A. F. Diaz, J. I. Castillo, J. A. Logan, and W. Y. Lee, J. Electroanal. Chem., 129, 115 (1981).

20. S. Dong and J. Ding, Synth. Metals, 20, 119 (1987).

21. V. Bocchi and G. P. Gardini, J. Chem. Soc., Chem. Commun., 148 (1986).

22. I. Ikemoto, K. Kikuchi, K. Yakushi, and H. Kuroda, Solid State Commun., 42, 257 (1982).

23. Ref 10, p 304. 